\title{
Effective elastic moduli of nanocomposites with prescribed random orientation of nanofibers
}

\author{
V. A. Buryachenko*, A. Roy** \\ *University of Dayton Research Institute, \\ OH 45469-0168, USA, Valeriy.Buryachenko@wpafb.af.mil \\ **Air Force Research Laboratory, AFRL/MLBC, \\ Wright-Patterson AFB, OH 45433-7750, USA, Ajit.Roy@wpafb.af.mil
}

\begin{abstract}
Nanocomposites are modeled as a linearly elastic composite medium, which consists of a homogeneous matrix containing a statistically homogeneous random field of nanofiers with prescribed random orientation. Estimation of effective elastic moduli of nanocomposites was performed with the effective field method (MEF, see for references and details [1]) developed in the framework of quasi crystalline approximation when the spatial correlations of inclusion location take particular ellipsoidal forms called "correlation hole". The parametric numerical analyses revealed that the most sensitive parameters influencing of the effective moduli are the axial elastic moduli of nanofibers rather then their transversal moduli, justified selection of correlation holes, concentration and prescribed random orientation of nanofibers.
\end{abstract}

Keywords: A. microstructures, B. inhomogeneous material, B. elastic material, B. nanocomposites.

\section{INTRODUCTION}

The recent discovery of carbon nanotubes (CNTs) has gained ever-broaded interest due to providing unique properties generated by their structural perfection, small size, low density, high strength, and excellent electronic properties. Indeed, the longitudinal Young's modulus of CNTs falls between 0.4 and 4.15 TPa, and a tensile strength approaching 100GPa. Experimental research and molecular dynamic simulation indicated that nanofibers can be effectively considered in the framework of continuum mechanics as the homogeneous prolate spheroidal anisotropic homogeneous inclusions (see [2]) with a large aspect ratio $(\alpha \approx 1000)$. In this paper, a nanocomposite is modeled as a linearly elastic composite medium, which consists of a homogeneous matrix containing a statistically homogeneous random field of nanofibers $v_{i}$ with prescribed random orientation described by the orientation distribution function (ODF). The prediction of the behavior of composite materials by the use of mechanical properties of the constituents and their microstructure is a primary problem of micromechanics which can ultimately lead to the estimation of stress fields in the constituents. The quantitative description of the microtopology of heterogeneous media, such as fiber composite materials, is crucial in the prediction of overall mechanical and physical properties of these materials. For example, many studies have shown that both tensile ductility and fracture properties of multi-phase composite materials are strongly affected by the spatial heterogeneity of the reinforcing phases (see for references e.g. [1]). Even after many years of comprehensive study by extremely laborious direct measurements and empirical relations, the effect of the structure of microinhomogeneous materials and their influence on the mechanical properties is not completely clear.

\section{ESTIMATION OF EFFECTIVE MODULI OF NANOCOMPOSITES}

Estimation of effective elastic moduli of nanocomposites was performed by the means of effective field method (MEF, see for references and details [1]) based on a so-called effective field hypothesis: each fiber is embedded in the homogeneous effective field depending on prescribed random orientation of this fiber [in contrary to the Mori-Tanaka method (MTM) where the effective field coincides with the average stresses in the matrix]. It may be noted the certain drawbacks of the extension of MoriTanaka method to multiphase composites: Mori-Tanaka moduli may violate the Hashin-Shtrikman bounds and are non-symmetric for the general diphase composites. In the authors knowledge, no models exists that satisfies all of theoretical criteria mentioned above for arbitrary phase anisotropy and fiber-orientation distributions. With the rapid development and increasing application of multyphase composites, especially the composites reinforced by the nanofibers, a micromechanical approximation devoided of the mentioned contradictions is highly desirable.

MEF was used in the framework of quasi crystalline approximation when the spatial correlations of inclusion location called "correlation hole" take particular ellipsoidal forms. This "correlation hole" $v_{i}^{0}$, enclosing the representative fiber $v_{i}$, does not occupy the centers of surrounding fibers (since they cannot overlap) and compatible with mutual orientations of fibers. The independent justified choice of shapes of inclusions and 
correlation holes provides the formulae of effective moduli which are completely explicit and easy to use. If the shapes of fibers $v_{i}$ and the correlation holes $v_{i}^{0}$ coincide, then the estimations of the effective moduli $L^{*}$ are symmetric and coincide for both the arbitrary random field of isotropic fibers and aligned anisotropic fibers. However, the main advantage of the proposed approach is that it is free from some of drawbacks of other approximation such as the Mori-Tanaka scheme, which can generate tensors of effective moduli which fails to satisfy a necessary symmetry requirement. Most significantly, the MEF's estimations are found to be in disagreement with corresponding estimations by the MTM in the case of the randomly oriented anisotropic inclusions in the composites. The current approach takes into account both the anisotropic moduli of fibers and specific shape of the correlation holes $v_{i}^{0}$ reflecting the anisotropy of ODF that was not considered in the past (see, e.g., [3]). The most sensitive parameters influencing on the effective moduli are the axial elastic moduli of nanofibers rather then their transversal moduli, as well as the justified choice of the shape correlation holes, concentration and prescribed random orientation of nanofibers.

In the parametric analysis we considered both isotropic (see [4]) and anisotropic fibers (see [2]) in the Epon 862 matrix (see [5]). Three random orientations of fibers were considered: aligned fibers parallel to the axis $O x_{1}$ (1D uniform random orientation), uniform plane random orientation of fiber parallel to the plane $\mathrm{Ox}_{1} x_{2}$ (2D uniform random orientation), and 3D uniform random orientation. The effective elastic Young's moduli $E^{*}$ and experimental data by Lafdi and Matzek [6] vs the fiber volume concentrations $c$ of the isotropic and anisotropic fibers with $3 \mathrm{D}$ uniform random orientation are presented in Fig. 1.

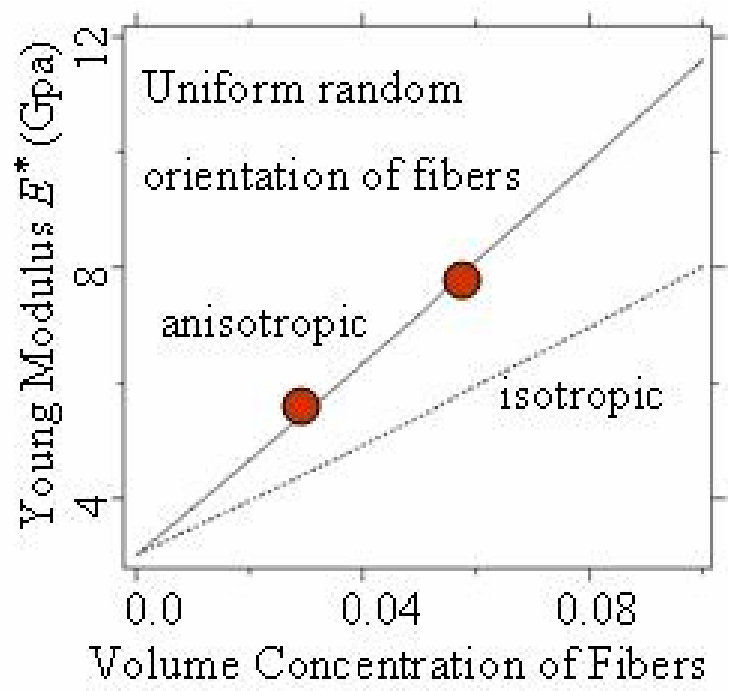

Fig. 1. Modulus $E^{*}(\mathrm{GPa})$ vs $c$
In the case of 3D uniform random orientation of nanofibers, the tensors of the effective elastic moduli $L^{*}$ obtained by the MTM and MEF are symmetric and coincide in cases with the same domain shapes $v_{i}$ and $v_{i}^{0}$ for both the isotropic and anisotropic fibers. It can be seen that Young's modulus $E^{*}$ estimated for anisotropic fibers is in 1.5 times stiffer than $E^{*}$ for the isotropic fibers, although the transversal Young modulus $E_{3}$ of anisotropic fibers is 30 times softer than $E_{3}$ for the isotropic fibers. However, for 2D uniform random orientation of fibers, the MTM generates the tensors of effective moduli which fail to satisfy a necessary symmetry requirement (see Fig. 2).

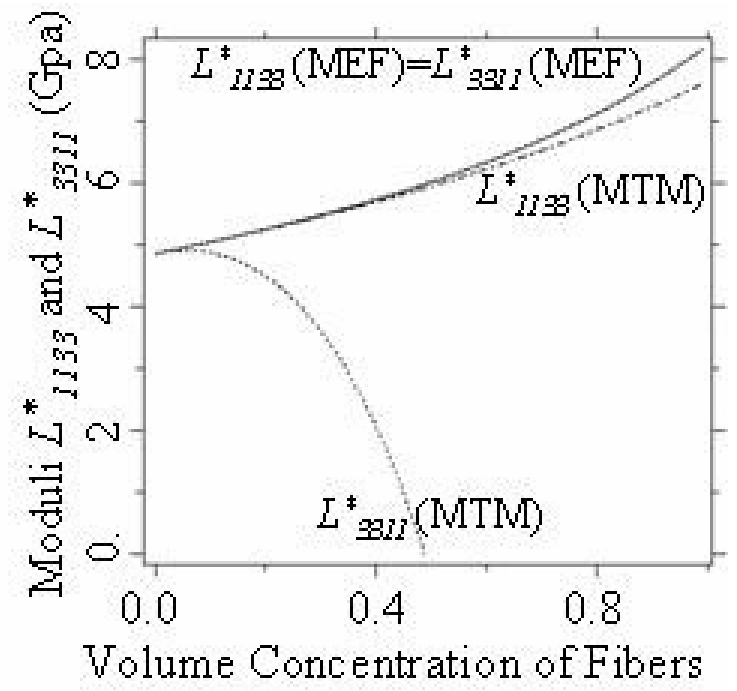

Fig. 2. $L^{*}{ }_{1133}$ and $L^{*}{ }_{3311}(\mathrm{GPa})$ vs $c$

To avoid of the deficiency in the case of 2D uniform random orientation of nanofibers, the MEF was applied with the reasonable shape of "correlation hole" $v_{i}^{0}$ in the form of oblate spheroid (with aspect ratio 1/ $\alpha$ ) with big

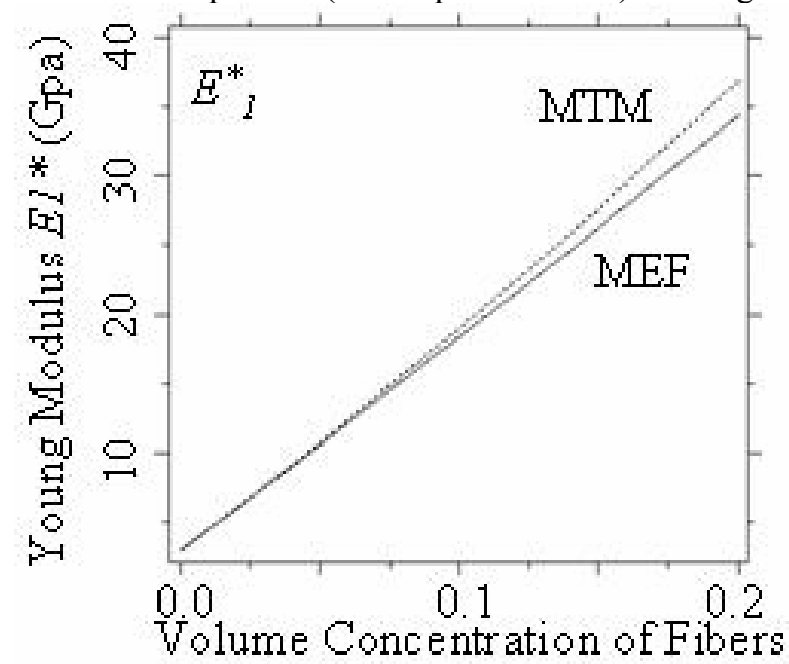

Fig. 3. $E^{*}(\mathrm{GPa})$ vs $c$ 
semiaxes parallel to the plane $O x_{1} x_{2}$. We can make the following conclusions. The estimations of the effective elastic moduli by the MEF and by MTM are different for any nonzero components of the tensor $L^{*}{ }_{i j k l}$. The matrix of effective moduli estimated by the MEF is symmetric $\left(L^{*}{ }_{i j k l}=L^{*}{ }_{k l i j}\right)$ in all investigated diapason of a fiber concentration $c$, and their elastic moduli. The diagonal elements of the matrixes $L^{*}{ }_{i j k l}$ estimated by the MEF and by the MTM agree well at $c<0.5$ (for example, the Young's moduli $E^{*}{ }_{1}$ and $E^{*}{ }_{3}$ estimated by both the MEF and MTM are very close, see Figs. 3 and 4), however, the nondiagonal elements can differ by a great extent for $c>0.2$. In so doing the matrix $L^{*}{ }_{i j k l}$ estimated by the MTM is not symmetric $\left(L^{*}{ }_{i j k l} \neq L^{*} k l i j\right.$, see Fig. 2$)$.

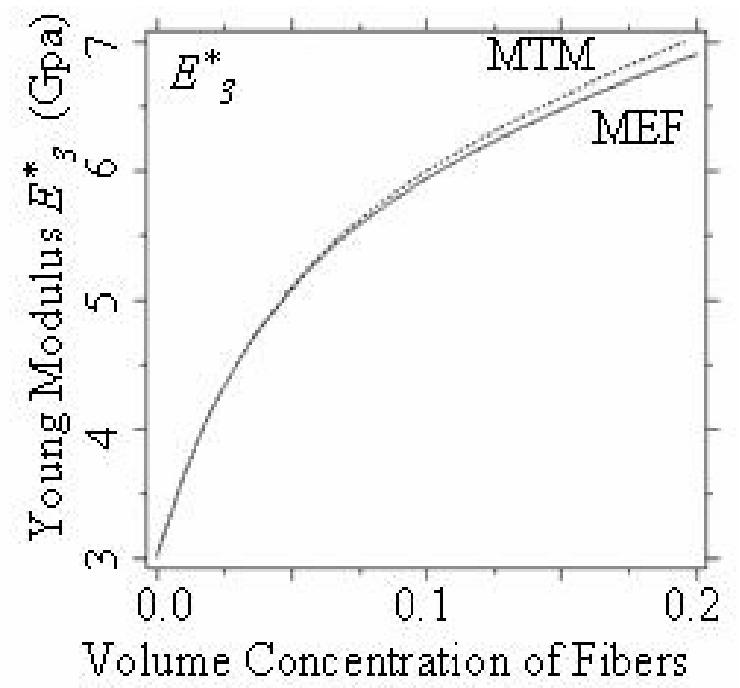

Fig. 4. $E^{*}{ }_{3}(\mathrm{GPa})$ vs $c$

\section{CONCLUDING REMARCS}

Based on the MEFM method, a micromechanical model has been developed to predict the effective moduli of nanocomposites reinforced by the anisotropic nanofibers with prescribed orientation. The model proposed in the present paper is able to analyze the effect of fiber orientations on the effective elastic moduli of composites. The current approach takes into account both the anisotropic moduli of fibers and specific shape of the correlation holes $v_{i}^{0}$ reflecting the anisotropy of ODF that was not considered in the past (see [3]).

The independent justified choice of shapes of inclusions and correlation holes provides the formulae of effective moduli which are completely explicit and easy to use, and depend on two microstructural tensors characterizing the shape of fibers and their distribution, respectively. If the shapes of fibers $v_{i}$ and the correlation holes $v_{i}^{0}$ coincide, then the estimations of the effective moduli $L^{*}$ are symmetric and coincide for both the arbitrary random field of isotropic fibers and aligned anisotropic fibers. However, the main advantage of the proposed approach is that it is free from some of drawbacks of other approximation such as the Mori-Tanaka scheme, which can generate tensors of effective moduli which fails to satisfy a necessary symmetry requirement. Most significantly, the MEF's estimations are found to be in disagreement with corresponding estimations by the MTM in the case of 2-D uniformly randomly oriented anisotropic fibers in the composites. The most sensitive parameters influencing on the effective moduli are the axial elastic moduli of nanofibers rather then their transversal moduli, as well as the justified choice of the shape correlation holes, concentration and prescribed random orientation of nanofibers.

The results of the parametric analyses lead to the following conclusions:

1) Effective stiffness of nanocomposites is defined by the axial stiffness of fibers rather than their transversal stiffness.

2) The variation of fiber orientation is critical and can lead to the variation of the effective stiffness of nanocomposites as much as times for the fiber volume concentration $c<0.1$.

3) The estimation method of the effective stiffness of nanocomposites is critical. For the plane uniform orientation of anisotropic fibers, the use of the Mori-Tanaka method is fundamentally inappropriate in contrast to the method of effective field.

\section{REFERENCES}

[1] Buryachenko, V.A. (2001) Multiparticle effective field and related methods in micromechanics of composite materials. Appl. Mech. Review 54(1), 1-47.

[2] Odegard, G. M., Gates, T. S., Wise, K. E., Park, C. and Siochi, E. J. (2003) Constitutive modeling of nanotubereinforced polymer composites. Composites Science and Technology, 63, 1671-1687.

[3] Ponte Castaneda, P. and Willis, J. R. (1995) The effect of spatial distribution on the effective behavior of composite materials and cracked media. J. Mech. Phys. Solids. 43, 1919-1951.

[4] Tibbetts, G. G. and McHugh, J. J. (1999) Mechanical properties of vapor-grown carbon fiber composites with thermoplastic matrices. J. Mater Res. 14, 28712880.

[5] Tandon, G. P., Kim, R. Y. and Rice, B. P. (2002) Influence of vapor-grown carbon nanocomposites on thermomechanical properties of graphite-epoxy composites. Proc. American Society for Composites $17^{\text {th }}$ Technical Conference, Perdue University, West Lafayette, Indiana, Paper 2039.

[6] Lafdi, K. and Matzek, M. (2003) Carbon nanofibers as a nano-reinforcement for polymeric nanocomposites. The 35th International SAMPE Technical Conference, Dayton, Ohio. 